\title{
The prevalence of unrecognized congenital heart disease among healthy elementary school students in northern Thailand
}

\author{
Jarun Sayasathida, Pentip Supachokchaipattana ${ }^{\mathrm{b}}$, Kraminee Pipatvechc, Kanchapan Sukonpan ${ }^{\mathrm{d}}$, \\ Naraporn Somboonna ${ }^{\mathrm{e}, \mathrm{f}}$, Supasit Pannarunothai ${ }^{\mathrm{g}}$ \\ ${ }^{a}$ Cardiac Center, Naresuan University Hospital, Phitsanulok 65000; ${ }^{b}$ Pediatric Department, \\ Sawanpracharak Hospital, Nakhon Sawan 60000; 'Pediatric Department, Uttaradit Hospital, \\ Uttaradit 53000; 'Obstretic and Gynecology Department, Buddhachinaraj Hospital, Phitsanulok 65000; \\ ${ }^{e}$ National Center for Genetic Engineering and Biotechnology, National Science and Technology \\ Development Agency, Pathumthani 12120, fDepartment of Microbiology, Chulalongkorn University, \\ Bangkok 10330; ${ }^{g}$ Faculty of Medicine, Naresuan University, Phitsanulok 65000, Thailand
}

\begin{abstract}
Background: Congenital heart disease (CHD) is one of the major congenital anomalies among newborns. Although many infants with CHD are symptomatic and identified soon after birth, others remain undiagnosed until the disease becomes severe.

Objectives: Unravel the number of CHD cases among 7-12 years old students who reported neither clinical complication nor history about cardiac diseases, stressing the importance for regular cardiac examination in children, even if they appeared healthy.

Methods: The study selected students without clinical history of heart disease from 1,041 elementary schools in northern Thailand (Tak, Nakhon Sawan and Uttaradit). Certified nurses and health officers examined the students for abnormal heart conditions. Pediatric cardiologists scrutinized the students with suspected CHD for confirmation and characterization of cardiac lesion types.

Results: Out of 144,293 students included in the study, 851 students showed atypical heart symptoms, and 87 were verified to have the CHD. This conferred the prevalence rate of unrecognized CHD among the elementary school children to be 0.60 per 1,000. The frequently-detected heart defects were ventricular septal defect (41.4\%) and pulmonary stenosis (16.1\%). Fifty-one children (58.6\%) required surgery for cardiovascular correction. The prevalence of the CHD in elementary school students ranged from 0.41 to 1.05 cases per 1000 study subjects. The greatest number of incidences was found in Tak province where medical resources are inadequate.

Conclusion: This study highlights the significance of routine cardiac examination among school-aged students, particularly in the regions with limited medical facilities, providing information essential for appropriate health care planning and managements.
\end{abstract}

Keywords: Asymptomatic, congenital, elementary school students, heart defect, heart disease, prevalence

Congenital heart disease (CHD) is one of the major problems affecting the public health worldwide. CHD not only causes high morbidity and mortality in infants, but also affects the quality of life in the childhood and, later, in the adulthood. The occurrence

Correspondence to: Jarun Sayasathid, MD. Cardiac Center, Naresuan University Hospital Phitsanulok 65000, Thailand. Email: jsayasathid@hotmail.com of congenital malformation of the heart and the large vessels ranges from 3.7 to 17.5 per 1000 live births in the world [1-3], accounting for approximately $30-40 \%$ of all congenital defects $[4,5]$.

Children with a critical cardiac lesion showed high morbidity and mortality during childhood. While many newborns with the cardiac disorder are symptomatic and identified soon after birth, many others are not diagnosed until later. The risk of morbidity and mortality 
increased as the delay in diagnosis and treatment occurred [6].

The current reports on incidences of the CHD in live births in Thailand were inconsistent. The variation among the reports might be due to a single or combination of the following factors, including methodologies that were used to diagnose the CHD, skills and techniques of the examiners, and ethnic and regional backgrounds of the examinees [7-9]. Additional factors included problems associated with retrospective studies in which the data on previous medical records, defective registration, lack of congenital malformation registry, lack of coordination between cardiac pediatricians in outpatient and private clinics, and the absence of an autopsy procedure to determine some fatal incidences among the stillborns [1-3, 7-9].

The present study aimed to uncover the prevalence of children with unreported CHD, and allow them to undergo treatment before the diseases would become severe and untreatable. To properly identify the rate of unrecognized CHD, we used a prospective study, specially trained all cardiac examiners, selected the subjects from similar age range (7-12 years old), and classified the data based on regional backgrounds and types of cardiac lesions.

\section{Materials and methods}

A prospective study was performed in three provinces in the lower North of Thailand between January 2006 and September 2008, and 144,293 healthy students from 1,041 elementary schools in Tak, Nakhon Sawan, and Uttaradit provinces were included. Students with a history of cardiovascular malformation or cardiac surgery prior to the beginning of the study, rheumatic heart disease, heart arrhythmia without structural cardiac abnormalities, and who were absent from the school on the scrutiny day were excluded from the study.

We used the cardiac investigation procedures as shown in Fig. 1. The primary screening comprises of physical and cardiovascular examinations on all the subjects to examine the students with potential heart defects, including heart arrhythmia, heart murmur and cyanosis. The primary screening was performed by 457 qualified nurses and health officers. For the effective primary screening, all of the qualified health care professionals were trained and passed the tests for the heart defect diagnosis by pediatric cardiologists with the scores of at least $90 \%$. Following the first screening step, the students with abnormal heart condition were referred to the pediatric cardiologists for complete cardiovascular examination and treatment. The final diagnostic step authenticated the students with the CHD, and identified the different types of congenital heart defects using electrocardiogram, echocardiography, and chest $\mathrm{X}$-ray.

The data were collected and analyzed to determine the prevalence, as well as the correlation between the primary screening and the diagnosis by physician. The prevalence of unrecognized CHD was calculated by the number of students with unrecognized CHD divided by the total number of students who were enrolled in the study.

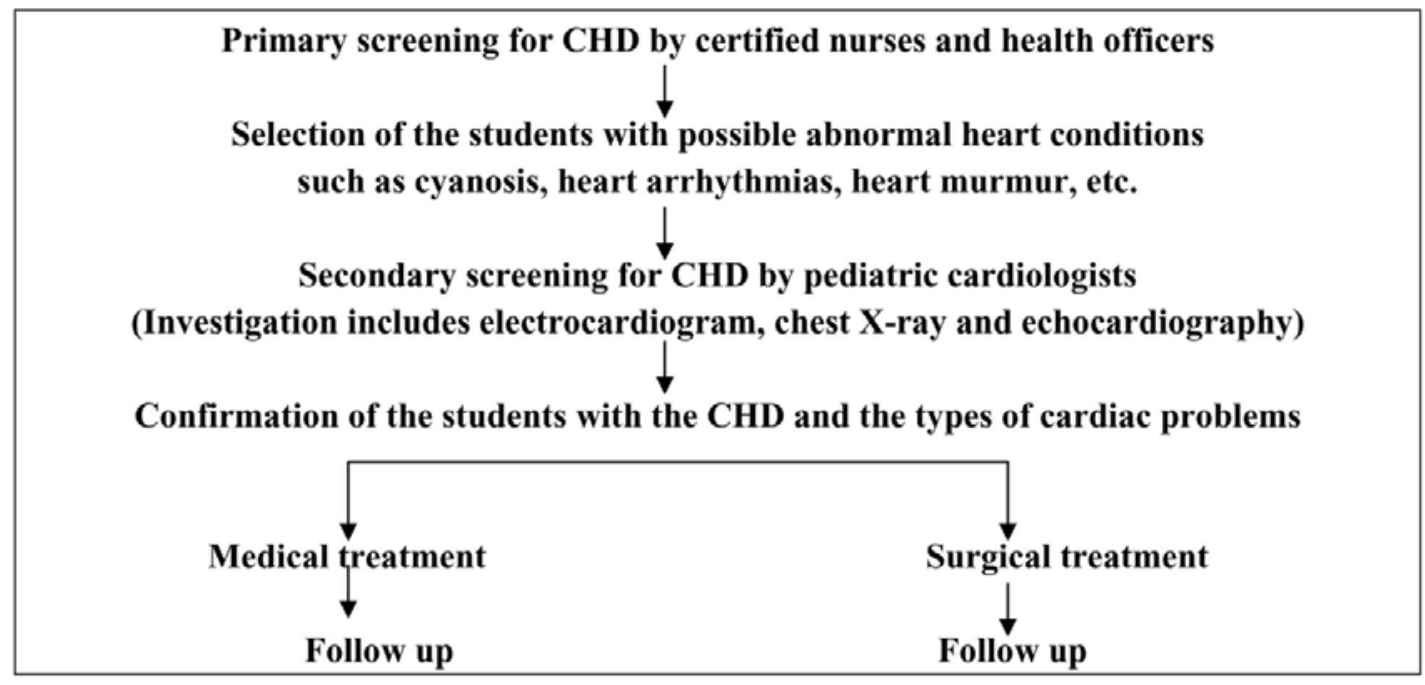

Fig. 1. Research flow chart starting from primary screening to treatment of CHD. 


\section{Results}

From 1,041 elementary schools of three southern provinces of northern Thailand, 144,293 students had no history of CHD. Following the primary screening, 851 students (275 from Tak, 492 from Nakhon Sawan, and 82 from Uttaradit) were suspected to have abnormal heart symptoms (Table 1). Heart diseases were confirmed by pediatric cardiologists in 98 children, inferring the overall effectiveness of the CHD detection by the primary screening to be $11.5 \%$ (14.6\% for Tak, $7.3 \%$ for Nakhon Sawan, $13.4 \%$ for Uttaradit). Yet, 11 students were considered to be nonCHD, because five students had rheumatic mitral regurgitation and six students had heart arrhythmias. Consequently, 11 non-CHD students were excluded from the data analyses, resulting in 87 confirmed cases of unrecognized CHD. The prevalence rate in the lower North of Thailand for the unrecognized CHD was 0.60 per 1000 elementary school-aged students. When classified by the provinces, the prevalence rates of unrecognized CHD in Tak, Nakhon Sawan and Uttaradit were $1.05,0.45$ and 0.41 , respectively.
The secondary cardiac investigation processes also defined types of abnormal heart conditions. Table 2 shows type of congenital heart problems detected among students.

The most frequently identified heart defects in this population were ventricular septal defect (VSD) (41.4\%), pulmonary stenosis (PS) (16.1\%), and patent ductus arteriosus (PDA) (12.6\%). Interestingly, VSD, PS, and PDA accounted for $70.1 \%$ of the total CHD. VSD accounted for approximately three folds higher than those of the PS and the PDA (2.57-fold for PS, 3.29-fold for PDA).

All the diagnosed CHD students received medical or surgical treatment, depending on the pediatric cardiologists' evaluation. ASD, VSD, PDA, and TOF were given respective priority for cardiac surgery, and the definitive cardiac operation for correction was performed in 51 children (58.6\%). All of the students who had heart operation in the study had no complication, and their qualities of life were reported improved after obtaining the treatment.

Table 1. The prevalence of congenital heart disease by provinces following primary and secondary screenings. Primary screening was performed by certified nurses and health staffs. Secondary screening was performed by specialized cardiologists, and investigation processes included electrocardiogram, chest X-ray and echocardiography.

\begin{tabular}{lccccc}
\hline Province & $\begin{array}{c}\text { Number of } \\
\text { schools }\end{array}$ & $\begin{array}{c}\text { Number of } \\
\text { students }\end{array}$ & $\begin{array}{c}\text { Students with } \\
\text { positive } \\
\text { screening }\end{array}$ & $\begin{array}{c}\text { Students with } \\
\text { confirmed } \\
\text { CHD }\end{array}$ & $\begin{array}{c}\text { Prevalence } \\
\text { rate (per 1,000 } \\
\text { children) }\end{array}$ \\
\hline Tak & 231 & 38,055 & 275 & 40 & 1.05 \\
Nakhon Sawan & 591 & 79,319 & 494 & 36 & 0.45 \\
Uttaradit & 219 & 26,919 & 82 & 11 & 0.41 \\
Total & 1041 & 144,293 & 851 & 87 & 0.60 \\
\hline
\end{tabular}

Table 2. Type of congenital heart problems detected among students in three southern provinces of northern Thailand.

\begin{tabular}{lccc}
\hline Cardiac lesion & \multicolumn{2}{c}{ Congenital heart disease } & Prevalence rate \\
\cline { 2 - 4 } & Total cases (n) & Percentage (\%) & (per 1,000 children) \\
\hline Ventricular septal defect (VSD) & 36 & 41.4 & 0.2495 \\
Pulmonary stenosis (PS) & 14 & 16.1 & 0.0970 \\
Patent ductus arteriosus (PDA) & 11 & 12.6 & 0.0762 \\
Atrial septal defect (ASD) & 8 & 9.2 & 0.0554 \\
Aortic stenosis (AS) & 6 & 6.9 & 0.0416 \\
Mitral valve prolapsed (MVP) & 5 & 5.7 & 0.0347 \\
Tetralogy of Fallot (TOF) & 3 & 3.4 & 0.0208 \\
Coronary arterio-venous fistula & 2 & 2.3 & 0.0139 \\
Tricuspid regurgitation (TR) & 1 & 1.1 & 0.0069 \\
Aortic regurgitation (AR) & 1 & 1.1 & 0.0069 \\
\hline Total & 87 & 100 & 0.6029 \\
\hline
\end{tabular}




\section{Discussion}

Several countries reported the CHD incidences among live births to be ranging from three to 17.5 per 1000. Particularly, pre-term infants generally have a prevalence rate two-three times higher than that of term infants [2]. The wide gap between the CHD rates may be attributable to a variety of factors, including genetics, environmental background, and methods and accuracy of the abnormal heart screening. Up to $75 \%$ of the children with CHD did not exhibit clinical signs of heart disease until the disease became severe. Hence, many children with CHD are unaware of their cardiovascular abnormality. This is a reason for the significant percentage of severe morbidity and mortality among elementary school students with no clinical symptoms. In contrast, approximately $25 \%$ of the cases show clinical symptoms and are treated, albeit the clinical symptoms in $15 \%$ of the cases would spontaneously recover within one year without treatment and surgery $[6,10]$.

A study in Egypt reported the CHD incidences among school children to be 1.01 per 1,000 students (881 cases in 869,434 students). Similar to the present results (Table 2) and many other studies [6, 11, 12], the most common cardiac defects were VSD and PS. Further more, studies based on children of Sukhothai and Khon Kaen provinces of Thailand revealed the prevalence rate of CHD to be 1.19 and 0.9 per 1,000 students, respectively $[6,12]$. According to these studies, half of the children with CHD showed distinct symptoms, such as exhaustion, retarded growth, and cyanosis.

The present study identified the average prevalence of unrecognized CHD among elementary school students to be 0.6 per 1000 (Table 1). The relatively fewer number of diagnosed CHD compared with other studies might be because, unlike other studies, this study only selected the students who reported healthy and had neither history nor complication regarding abnormal cardiac conditions. Hence, the students enrolled in this study indeed represented the healthy students. Meanwhile, the actual incidences of CHD among 7-12 years old children should be higher, depending on the number of students who reported CHD or cardiac symptoms prior to the age of seven years.

The relatively low prevalence of CHD in Nakhon Sawan and Uttaradit, compared with Tak (Table 1), might be due to the topography of Tak. It is surrounded by the mountains and the province itself has limited medical staffs and facilities. Consequently, the greater prevalence of the students with the CHD might not reflect the greater incidence of the CHD among live births in Tak, but a lack of CHD detection prior to the age of seven.

The gap between the actual number of unrecognized CHD confirmed by the cardiologists and the number referred from the primary screening (Table 1) may be explained by the miscommunication between the health officers and the students leading to the misinterpretation of the innocent cardiac murmur. The skills of the heath care staffs were also necessary. To reduce screening errors, initial relaxation to reduce the students' anxiety was recommended. Moreover, the longer period of staff training and practice will improve competency in CHD screening.

In conclusion, this study demonstrated the capacity of a health care system to discover unrecognized congenital heart defects in children. Comparative studies helped clarifying the distribution of CHD in various parts of Thailand. A better understanding of the distribution of CHD will allow health care planners to focus attention on why an elevated number of cases exist and how to alleviate the problem. In addition, analyses of genetic patterns associated with the greater risk for cardiovascular diseases in population from certain region may be conducted to help elucidating the association between the risk of heart abnormality and the ethnic background.

\section{Acknowledgements}

We like to thank Professor Boonchob Pogpanich for his support. We acknowledge nurses and health officers of Tak, Nakhon Sawan, and Uttaradit Provincial Health Office, and thank Dr. Mark Pinsker for his proofreading the manuscript.

This study was supported by the Cardiac Children Foundation of Thailand under the Royal Patronage of H.R.H. Princess Galyani Vadhana Krom Luang Naradhiwas Rajanagarindra. The authors have no conflict of interest to declare.

\section{References}

1. Bolisetty S, Daftary A, Ewald D, Knight B, Wheaton G. Congenital heart defects in central Australia. Med J Aust. 2004; 180:614-7.

2. Ferenez C, Rubin JD, McCarter RJ, Brenner JI. Congenital heart disease: prevalence at live birth. The 
Baltimore-Washington infant study. Am J Epidemiol. 1985; 121:31-6.

3. Kapoor R, Gupta S. Prevalence of congenital heart disease, Kanpur, India. Indian Pediatr. 2008; 45:309-11.

4. Romano-Zelekha O, Hirsh R, Blieden L, Green MS, Shohat $\mathrm{T}$. The risk for congenital heart defects in offspring of individuals with congenital heart defects. Clin Genet. 2001; 59:325-9.

5. Zierler S. Maternal drugs and congenital heart disease. Obstet Gynnecol. 1985; 65:155-65.

6. Mongkolsiri D, Taytiwat P, Pankat P. The prevalence of congenital heart disease of elementary school aged students in Sukhothai province. Thai Med Counc Bull. 2005; 34:91-104.

7. Begic H, Tahirovic H, Mesihovic-Dinarevic S, Ferkovic $\mathrm{V}$, Atic N, Latifagic A. Epidemiological and clinical aspects of congenital heart disease in children in Tuzla Canton, Bosnia-Herzegovina. Eur J Pediatr. 2003; 162: 191-3.
8. Grech V, Agius-Muscat H, Savona-Ventura C, Pace J. Regional differences in birth prevalence of congenital heart disease in Malta. Cardiol Young. 1999; 9:150-4.

9. Khalil SI, Gharieb K, Haj ME, Khalil M, Hakiem S. Prevalence of congenital heart disease among schoolchildren of Sahafa town, Sudan. Sudan Med J. 1997; 3:24-8.

10. Mitchell, Korones SB, Berendes HW. Congenital heart disease in 56109 births: incidence and natural history. Circulation. 1971; 43:323-9.

11. Bassili A, Mokhtar SA, Dabous NI, Zaher SR, Mokhtar MM, Zaki A. Congenital heart disease among School children in Alexandria, Egypt: an overview on prevalence and relative frequencies. J Trop Pediatr. 2000; 46:357-62.

12. Sayasathid J, Tantiwongkosri K, Somboonna N. Unrecognized congenital heart disease among Thai children. J Med Assoc Thai. 2008; 92:356-9. 